\section{PTU-080 CHANGE IN DISEASE ACTIVITY AND RESTING ENERGY EXPENDITURE IN CHILDREN WITH CROHN'S DISEASE}

doi:10.1136/gut.2011.239301.208

A EWiskin, ${ }^{1 *}$ S A Wootton, ${ }^{1}$ R M Beattie ${ }^{2}$ 'NIHR BRU (NUTRITION, DIET \& LIFESTYLE);

${ }^{2}$ Paediatric Medical Unit, Southampton General Hospital, Southampton, UK

Introduction Many children with Crohn's disease have poor nutritional status. Inflammation is thought to place a metabolic burden on the body which can be detected by raised resting energy expenditure (REE). As a result, increased REE unmatched by dietary intake is frequently implicated as a cause of weight loss, poor nutritional status and growth failure. In two separate cross-sectional studies we failed to demonstrate a relationship between REE and disease activity in children with Crohn's disease. We have performed an initial analysis of longitudinal data to assess changes in REE with disease activity in children with Crohn's disease.

Methods Children with Crohn's disease recruited from the regional paediatric gastroenterology unit were studied on two occasions at least 6 months apart. REE was measured by indirect calorimetry, fat free mass (FFM) was derived from weight measurement and estimates of fat mass from skinfold thicknesses. Disease activity was measured using the Paediatric Crohn's Disease Activity Index (PCDAI) and serum CRP.

Results 18 children were studied with a mean of $1.6 \pm 0.6$ years between measurements. Children showed mean increases in height, weight and BMI standard deviation scores of $0.1,0.5$ and 0.7 . PCDAI decreased by a mean of $6.5 \pm 16$ points. CRP decreased by a mean of $7 \pm 30 \mathrm{mg} / \mathrm{L}$. Mean fat free mass increased from $32.6 \mathrm{~kg} \pm 9.8$ to $39.7 \mathrm{~kg} \pm 11.4$ ( $\mathrm{p}=0.048)$. REE increased from $1379 \mathrm{kcal} \pm 293$ to $1522 \mathrm{kcal} \pm 297$ ( $\mathrm{p}=0.14)$. REE $/ \mathrm{kg} \mathrm{FFM}{ }^{0.5}$ was unchanged, $243 \pm 21$ and $244 \pm 28$.5. There was no obvious relationship between change in REE/ $/ \mathrm{kgFM}^{0.5}$ and change in PCDAI $r=-0.37$; $\mathrm{p} 0.12$ between the two time points.
Conclusion Cross-sectional studies of REE and disease activity in children with Crohn's fail to demonstrate a relationship between energy expenditure and disease activity when body size is accounted for. Using children studied twice we have failed to demonstrate any association between change in REE with change in disease activity, over time. A larger study is needed to determine this prospectively.

Competing interests None.

Keywords inflammatory bowel disease, paediatrics, resting energy expenditure. 